\title{
A COMPARISON OF TWO ACTUATORS FOR A SEMI-ACTIVE HELMHOLTZ RESONATOR
}

\author{
Charles B. Birdsong \\ Department of Mechanical Engineering \\ Michigan State University \\ East Lansing, MI 48824-1229
}

\section{ABSTRACT}

Two electro-mechanical actuators are examined for a semiactive Helmholtz resonator acoustic device. The device is used to reflect narrow band noise back to the source in an acoustic duct. The controller and actuator are used to tune the system on-line allowing optimum performance over a range of operating conditions. Actuator dynamics play an important role in the controller design and the operation of the device. Two variations of an electro-mechanical actuator are considered here. The first uses a dual voice coil speaker with local feedback compensation and the second uses the same speaker without the compensation. It is shown that both arrangements are effective but with competing advantages. The compensated actuator provides more control authority but adds considerable background noise while the uncompensated actuator provides less control authority but adds no background noise. The choice of actuator depends on the noise control objectives of the particular application.

\section{INTRODUCTION}

The semi-active Helmholtz resonator (Birdsong, 1999) is an acoustic device with behavior that can be used to selectively quiet narrow band noise in acoustic systems. It consists of a static Helmholtz resonator with a sensor, controller, and actuator added to the interior of the resonator cavity (Figure 1). The nominal resonant frequency and damping of the device is determined by the dimensions of the resonator neck and cavity (Temkin, 1981) but can be modified by the closed loop feedback system. When driven by a pressure from a primary acoustic system, such as an acoustic duct, the resonator responds with a large magnitude volume velocity through the resonator neck, which is in phase with the pressure. This creates a "pressure release" boundary condition, which inverts and reflects the incident pressure wave back up the duct, thus reducing the transmitted pressure wave and reducing the transmitted sound

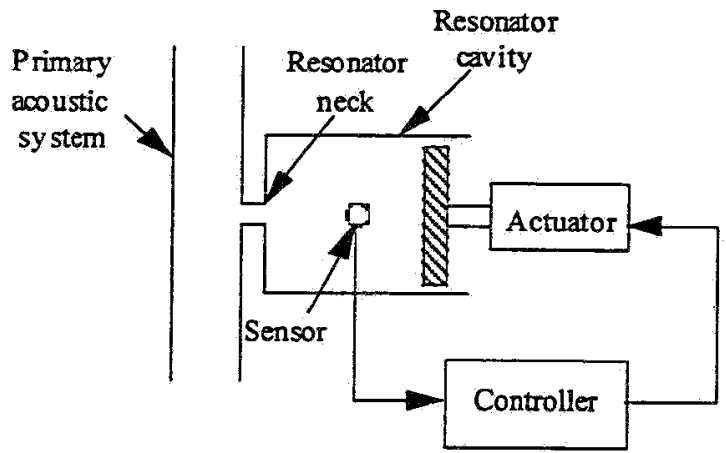

Figure 1. Schematic of a semi-active Helm holtz resonator connected to a primary acoustic system

\author{
Clark J. Radcliffe \\ Department of Mechanical Engineering \\ Michigan State University \\ East Lansing, M1 48824-1229
}

(Pierce, 1981).

The actuator is a critical component in the implementation of the SHR, and strongly effects the closed-loop performance of the system. Internal actuator dynamics will effect the closed-loop response of the system since the actuator and acoustic resonator become tightly coupled by the pressure interaction between the two systems. Electromechanical audio speakers are often used for acoustic actuators because of their low cost and commercial availability. However, audio speakers are not ideal actuators. They typically have a resonance frequency between $50-150 \mathrm{~Hz}$ (bass speakers) resulting in large magnitude and phase variation in their operating frequency range. Furthermore, the speaker velocity response is strongly effected by the pressure interaction with the acoustic system. A resonance in the acoustic system will impede the speaker velocity, resulting in weak control authority.

The closed-loop feedback control design for the SHR (Birdsong, 1999) is also effected by the actuator performance. A simple proportional-integral (PI) controller is used in the SHR, and an analytical solution can be found that maps the controller gains to the acoustic resonant frequency and damping ratio. However this assumes that the actuator has no dynamics, and the transfer function is a pure gain. Actuator dynamics complicate this mapping, resulting in the need of a higher order controller.

This sensitivity of the system response and the desire to avoid using a higher order controller motivates the use of local feedback compensation of the actuator. This technique adds a local feedback loop to the actuator (Figure 2), which drives the actuator output to the input signal, making the response approach a pure gain of one, as the loop gain is increased. The goal of the compensator is to boost the control authority. It also simplifies the controller design since the actuator response approaches the ideal response. Compensation for audio speakers has been proposed in many forms (Harwood, 1974; Klaassen and de Koning, 1968; Holdaway, 1963; Tanner, 1951). Birdsong and Radcliffe (1999) proposed a technique using a dual voice coil speaker with local feedback compensation that resulted in a compensated acoustic actuator with minimal magnitude and phase error below $400 \mathrm{~Hz}$. This design compensated the internal speaker dynamics and the pressure interaction with the acoustic system. The compensated acoustic actuator was chosen as the actuator for the SHR because of these strengths.

This paper discusses three major topics: analytical model development, coupled system simulations, and experimental results. In the first section, separate analytical models for each component are presented, including the acoustic resonator, controller, speaker, and compensator. In the second section, the models are coupled and various configurations are examined. First, the closed-loop resonator model with an ideal actuator is presented. Second, the ideal actuator is replaced by the compensated speaker model. Finally the uncompensated speaker model is applied to the closed-loop control system 


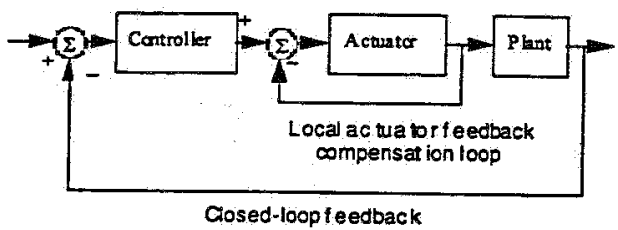

Figure 2. Local actuator feedback compensation used to boost actuator authority, minimize actuator dynamics, and simplify controller design

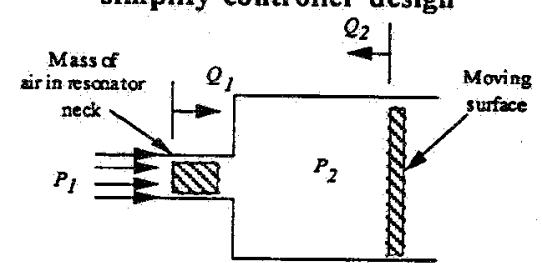

Figure 3. Schematic diagram of SHR showing inertia effect in neck and the movable surface in the cavity interior

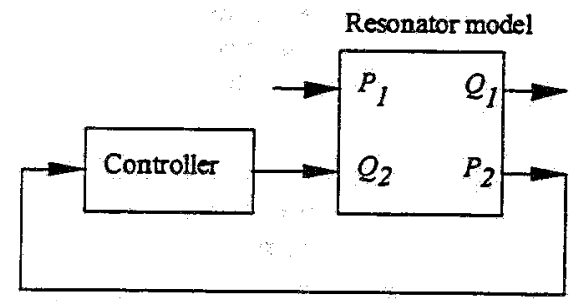

Figure 4. Closed-loop positive feedback SHR system block diagram with disturbance through $P_{1}$

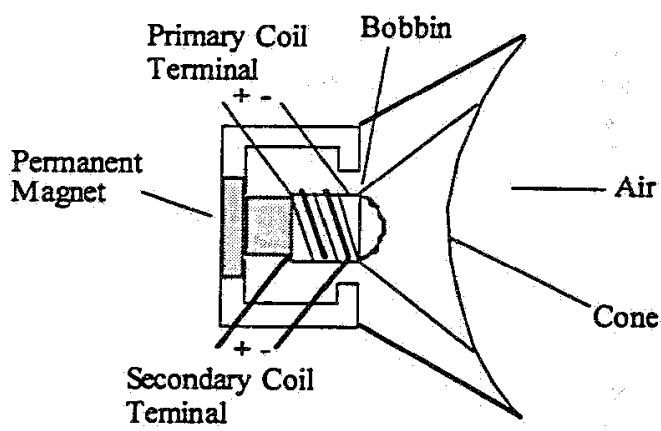

Figure 5. Dual voice-coil speaker diagram

demonstrating the advantages and disadvantages of the compensation technique. The last section presents experimental results which are compared with the analytical model and which demonstrate the effectiveness of the actuator implementation in the SHR.

\section{ANALYTICAL MODELDEVELOPMENT}

The closed-loop compensated SHR consists of four interconnected components: an acoustic resonator, a feedback controller, an audio speaker, and a compensator. Analytical models for each have been developed in other works, and will be presented here briefly. The reader is referred to the references for complete descriptions of the components. These component models will be assembled into coupled system models in the next section.

\section{Resonator}

The central component of the SHR is a Helmholtz resonator with one surface of the cavity replaced by a moving surface (Figure 3). The system can be represented by linear time invariant state equations (Birdsong, 1999)

$$
\begin{gathered}
\left.\frac{d}{d t}\left[\begin{array}{l}
Q_{1} \\
V
\end{array}\right]=\left[\begin{array}{cc}
\frac{-R_{a}}{I_{a}} & \frac{-1}{l_{a} C_{a}} \\
1 & 0
\end{array}\right] \begin{array}{c}
Q_{1} \\
V
\end{array}\right]+\left[\begin{array}{cc}
\frac{1}{I_{a}} & 0 \\
0 & 1
\end{array}\right]\left[\begin{array}{c}
P_{1} \\
Q_{2}
\end{array}\right] \\
{\left[\begin{array}{l}
Q_{1} \\
P_{2}
\end{array}\right]=\left[\begin{array}{cc}
1 & 0 \\
0 & \frac{1}{C_{\sigma}}
\end{array}\right]\left[\begin{array}{l}
Q_{1} \\
V
\end{array}\right]}
\end{gathered}
$$

where the states are $\mathcal{Q}_{1}$, the volumetric flow rate or "volume velocity" from the neck $\left(\mathrm{m}^{3} / \mathrm{s}\right)$ and $V$, the sum of the volumes introduced through the neck and the inner surface of the cavity $\left(\mathrm{m}^{3}\right)$. The inputs are $P_{1}$, the pressure at the neck inlet to the cavity $\left(\mathrm{N} / \mathrm{m}^{2}\right)$, and $Q_{2}$, the volume velocity from the movable surface in the cavity $\left(\mathrm{m}^{3} / \mathrm{s}\right)$. The outputs are $Q_{1}$ and $P_{2}$, the pressure in the cavity $\left(\mathrm{N} / \mathrm{m}^{2}\right)$. The other parameters are $R_{a}$, the acoustic loss that represents viscous and radiation losses $\left(\mathrm{Ns} / \mathrm{m}^{5}\right), I_{a,}$ the acoustic inertia of the mass of air in the resonator neck $\left(\mathrm{Ns}^{2} / \mathrm{m}^{5}\right)$, and $C_{a_{3}}$ the acoustic compliance of the cushion of air in the resonator cavity $\left(\mathrm{m}^{3} \mathrm{~N}\right)$.

With the movable surface held fixed, the system is a second order oscillator (Tang and Sirignano, 1973, Temkin, 1981) with resonant frequency and damping given by

$$
\begin{gathered}
\omega_{n}=\sqrt{1 / C_{a} I_{a}} \\
\zeta=\frac{R_{a}}{2} \sqrt{\frac{C_{a}}{I_{a}}}
\end{gathered}
$$

\section{Controller}

A proportional-integral controller can be used to generate an acoustic impedance between $Q_{2}$ and $R_{2}$, on the moving inner surface of the SHR cavity. This creates a closed-loop, positive feedback configuration (Figure 4).

A PI controller can be modeled by the transfer function,

$$
G(s)=\frac{Q_{2}}{P_{2}}=K_{P}+\frac{X_{I}}{s}
$$

where $K_{p}$, and $K_{I}$ are the proportional and integral gains respectively.

\section{Speaker}

The dual voice coil speaker (Figure 5) has certain characteristics that make it ideal for use as an acoustic actuator. It has 2 independent wire coils intertwined and wrapped around a bobbin that is allowed to slide over a permanent magnet. The state equations for a dual voice coil speaker can be represented by the linear time invariant state equations (Radcliffe and Gogate, 1996; Birdsong and Radcliffe, 1999)

$$
\frac{d}{d t}\left[\begin{array}{c}
Q_{2} \\
q_{2} \\
\lambda
\end{array}\right]=\left[\begin{array}{ccc}
\frac{-R_{s}}{I_{s}} & \frac{-1}{C_{s} I_{s}} & \frac{b S_{d}}{I_{c} I_{s}} \\
1 & 0 & 0 \\
\frac{-b l}{S_{d}} & 0 & \frac{-R_{m}-R_{c}}{I_{c}}
\end{array}\right]\left[\begin{array}{c}
Q_{2} \\
q_{2} \\
\lambda
\end{array}\right]+\left[\begin{array}{cc}
0 & 0 \\
0 & \frac{-S_{d}}{I_{s}} \\
1 & 0
\end{array}\right]\left[\begin{array}{l}
e_{p} \\
P_{2}
\end{array}\right]
$$

where the states are the volume velocity and volume displacement from the speaker $Q_{2}$, and $q_{2}$, and the electromagnetic flux in the speaker coil $\lambda$ The inputs are the primary coil voltage, $e_{p}$, and pressure on the speaker face, $P_{2}$. The output equation is given by 


$$
\left[\begin{array}{l}
e_{b s} \\
i_{p} \\
\underline{Q}_{2}
\end{array}\right]=\left[\begin{array}{ccc}
b\left(1-\frac{M_{c}}{I_{c}}\right) & 0 & \frac{-M_{c}}{I_{c}^{2}}\left(R_{c}+R_{m}\right) \\
0 & 0 & \frac{1}{I_{c}} \\
1 & 0 & 0
\end{array}\right]\left[\begin{array}{c}
Q_{2} \\
g_{2} \\
\lambda
\end{array}\right]+\left[\begin{array}{cc}
\frac{M_{c}}{I_{c}} & 0 \\
0 & 0 \\
0 & 0
\end{array}\right]\left[\begin{array}{l}
e_{p} \\
P_{2}
\end{array}\right]
$$

where the outputs are the voltage in the secondary coil, $e_{b s,}$ the current in the primary coil, $i_{p}$ and $Q_{2}$. The parameters in (6) and $(7)$ are the speaker face area, $S_{d}$, speaker inertia, $I_{s}$, speaker compliance, $C_{s}$, speaker friction, $R_{s}$ speaker coil resistance, $R_{c}$, speaker coil inductance, $I_{c}$, speaker coil mutual inductance, $M_{c}$, speaker electromechanical coupling factor, $b l$, and the primary coil current sensing resistance, $R_{m}$.

\section{Compensator}

The frequency response of the speaker can be improved with local feedback compensation. The volume velocity of the speaker, $Q_{2}$, is strongly affected by the dynamics of the speaker and the pressure input, $\boldsymbol{B}_{2}$. These effects will combine to create magnitude and phase variations in the primary coil voltage to speaker velocity response, $Q_{2} /{ }^{e}$. One method of reducing these unwanted effects is to apply a proportional feedback controller (Figure 6) resulting in the closed system,

$$
r_{s p k r}(s)=\frac{V_{s p k r}(s)}{V_{d}(s)}=\frac{K_{a m p} G_{s p k r}(s)}{1+K_{a m p} G_{s p k r}(s) H(s)}
$$

where $V_{s p k}$ is the speaker velocity, $V_{d}$ is the desired velocity, $G_{\text {sptr }}$ is the transfer function that relates the input voltage to speaker velocity, $K_{o m p}$ is an amplifier gain, and $H(s)$ is a velocity sensor. If the sensor transfer function is a real constant, $k$ over the controller bandwidth, then the closed loop transfer function, $T_{\text {spkr }}(s)$, will approach a constant, $1 / k$ with zero phase (Philips and Harbor, 1991). This compensation forces the speaker cone velocity $v_{\text {sph }}$ to accurately follow the desired velocity input. The speaker volume velocity, $Q_{2}$, is equal to the speaker area, $S_{d}$, multiplied by the speaker velocity, $v_{s p k r}$. The result is independent of the speaker dynamics and the input pressure provided that the sensor has a constant transfer function over the controller bandwidth.

As $K_{\text {amp }}$ is increased, the transfer function approaches $1 / H(s)$ and the magnitude and phase variations approach zero. This approach requires that the velocity of the speaker face can be measured. A speaker velocity sensor is therefore needed which accurately predicts the speaker velocity in the presence of speaker and plant dynamics.

The relation between the speaker velocity and the two other measurable outputs (the secondary coil voltage, $e_{b s}$ and the primary coil current, $i_{p}$ ) can be computed from (6) and (7) in terms of $e_{b s}$ and ${ }^{i} p$ yielding

$$
v_{s p k r}(s)=H_{b s} e_{b s}(s)-H_{p}(s) t_{p}(s)
$$

where $H_{b s}=1 / b l$ and $H_{p}(s)=s M_{d} d b l$.

The secondary coil voltage, $e_{b s}$, can be measured directly from the speaker coil. The primary coil current, $i_{p}$, can be determined from the voltage across a resistor, $R_{m}$ placed in series with the primary coil, while $H_{b s}$ is a pure gain $(1 / b l)$. The mathematically improper, differentiating transfer function, $H_{p}$, cannot be strictly realized exactly, but an approximation

$$
\hat{H}_{p}(s)=\frac{M_{c}}{b l}\left(\frac{p_{1} s}{s+p_{1}}\right)
$$

can be used, where $p_{I}$ is a pole location selected such that $\hat{H}_{p}(s)$ approximates $H_{p}(s)$ over the controller bandwidth. Feedback compensation can now be implemented using the signal from the velocity sensor to compute the error between the desired velocity and the sensor velocity and a proportional controller to drive the speaker velocity to the desired velocity

\section{COUPLED SYSTEM SIMULATION}

The system dynamics of the device can be investigated by coupling the component models and using numerical simulation. The simulation was performed using Matlab and Simulink software on a digital computer. This software allows state space and transfer function models to be interconnected in a single model to compute coupled system, time and frequency response graphs. The numerical values for the acoustic resonator and speaker parameters used in the simulation are given in Table 1. These values were measured from the physical devices used in the experimental results section and have been shown to be accurate (Birdsong and Radcliffe, 1999, Radcliffe and Gogate, 1992).

Table 1. SHR Model Parameter Values

\begin{tabular}{|l|l|l|l|}
\hline$b l$ & $2.45 \mathrm{~N} / \mathrm{A}$ & $R_{c}$ & $5.7 \mathrm{ohm}$ \\
\hline$c_{o}$ & $343 \mathrm{~m} / \mathrm{s}$ & $R_{m}$ & $10 \mathrm{ohm}$ \\
\hline$C_{s}$ & $0.000260 \mathrm{~m} / \mathrm{N}$ & $R_{s}$ & $3.745 \mathrm{~N} \mathrm{sec} / \mathrm{m}$ \\
\hline$I_{c}$ & $0.002 \mathrm{H}$ & $S$ & $0.000254 \mathrm{~m}^{2}$ \\
\hline$I_{s}$ & $0.0076 \mathrm{Kg}$ & $S_{d}$ & $0.0133 \mathrm{~m}^{2}$ \\
\hline$L_{c}$ & $0.010 \mathrm{~m}$ & $V$ & $0.002 \mathrm{~m}^{2}$ \\
\hline$M_{c}$ & $0.001 \mathrm{H}$ & $\rho_{o}$ & $1.18 \mathrm{Kg} / \mathrm{m}^{j}$ \\
\hline$S_{m i c}$ & $4 \mathrm{mV} / \mathrm{Pa}$ & & \\
\hline
\end{tabular}

\section{Resonator and Controller with Ideal Actuator}

The first coupled system model that will be considered is the acoustic resonator with a closed-loop feedback controller and an ideal actuator (Figure 7). This is a simple model that assumes that the actuator is ideal, i.e., it has a transfer function that is a pure gain of one. The cavity pressure, $P_{2}$, is fed to the controller and the controller output is fed into the resonator cavity volume velocity input, $Q_{2}$. The system can either be disturbed by the

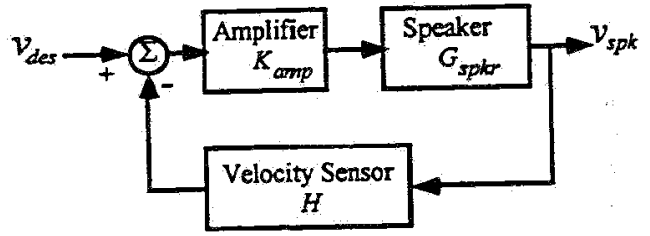

Figure 6. Block diagram of speaker and compensator

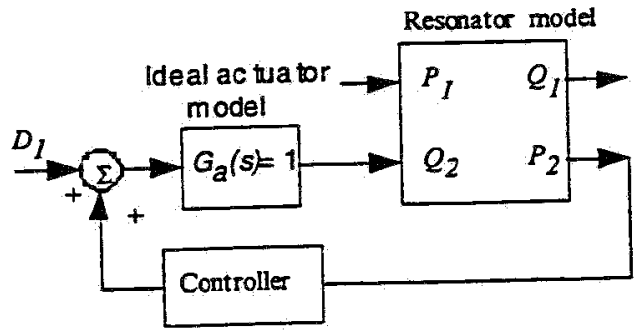

Figure 7. Block diagram of simple coupled system model including acoustic resonator, closed-loop feedback controller, and ideal actuator model 

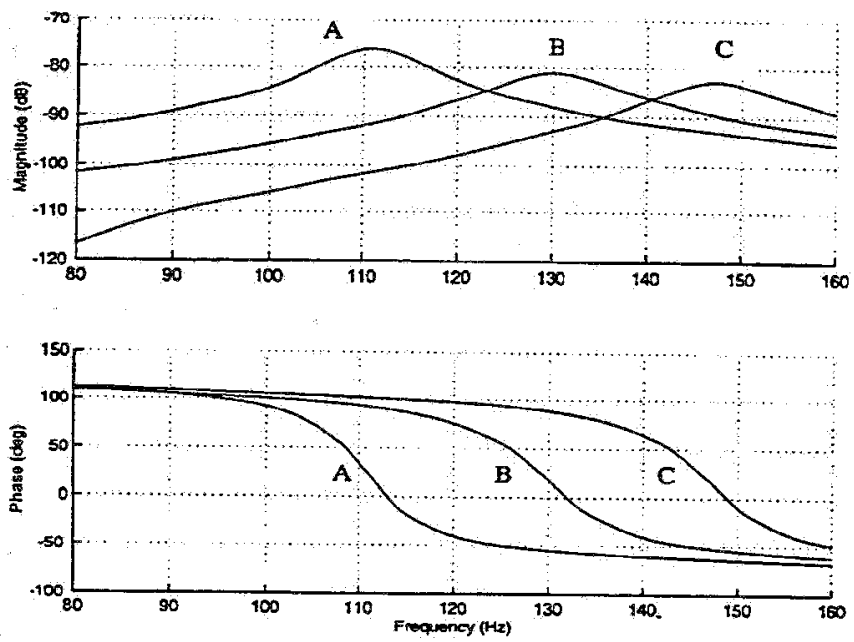

Figure 8. Frequency response simulation of resonator and closed-loop feedback controller with ideal actuator showing that the resonant frequency and damping can by changed by varying the controller gains (Table 2 ): $A: K_{P}=0.99, K_{I}=-100$, $B: K_{P}=0.99, K_{I}=0, C: K_{P}=0.99, K_{I}=100$

Table 2. Controller Gains used in Figure 8

\begin{tabular}{|c|c|c|c|c|}
\hline Graph & $K_{P}$ Gain & $K_{I}$ Gain & $\begin{array}{c}\text { Resonant } \\
\text { Freq. (Hz) }\end{array}$ & $\begin{array}{c}\text { Percent } \\
\text { Damping }\end{array}$ \\
\hline A & 0.99 & -100 & 112 & 10 \\
\hline B & 0.99 & 0 & 130 & 10 \\
\hline C & 0.99 & 100 & 145 & 10 \\
\hline
\end{tabular}

input, $P_{1}$, or by the disturbance signal, $D_{I}$, which is also added to the controller output.

The eigenstructure of the system can be modified with the positive feedback controller (Radcliffe, et. al., 1994). With the controller gains set to zero (open-loop), the system resonates at the nominal resonant frequency and damping (3) and (4). The numeric values for the resonator model nominal, resonant frequency and damping are $f_{n}=205 \mathrm{~Hz}$ and $\zeta=0.025$. By varying the controller gains $K_{P}$ and $K_{P}$, the resonant frequency and damping can be varied. Figure 8 shows the $P_{1} / Q_{1}$ transfer function for this model for various values of $K_{P}$ and $K_{I}$ (Table 2).

The feedback controller makes the system response appear identical to the response of three different passive Helmholtz resonators with different tuned frequencies. In each curve the magnitude attains a maximum at the same frequency that the phase crosses zero. This is identical to the response of a passive resonator. The important feature here is that the change in frequencies was created by electronic tuning, not by changing the physical dimensions of the resonator.

This system, with the ideal actuator model, can be used to compute an analytical solution that maps the PI controller gains, $K_{1}$ and $K_{p}$, to the closed-loop frequency response values of $\omega_{n}$ and $\zeta$. This is the basis for an adaptive control algorithm that changes the gains on line to tune the system to track a disturbance signal with slow time varying frequency (Birdsong, 1999). However, without the ideal actuator assumption, this mapping is not valid, and a different, more complicated controller design is required.

\section{Resonator, Speaker, Compensator, and Controller.}

The ideal actuator model can be replaced by the compensated speaker model, and the closed loop control of the resonator can be

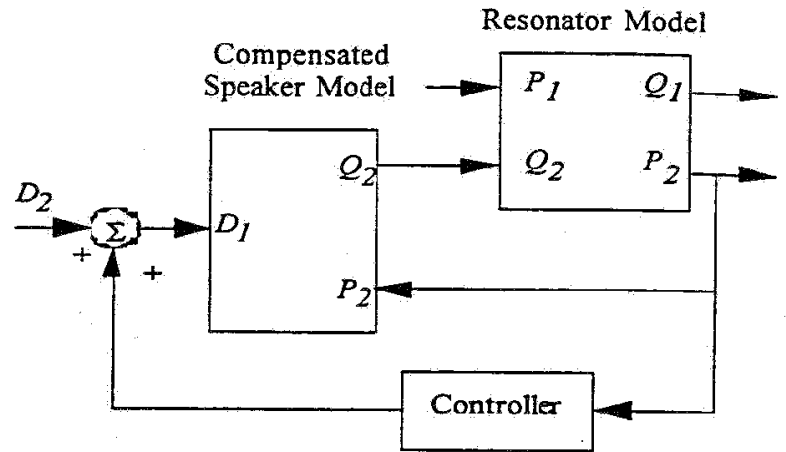

Figure 9. Block diagram of resonator, compensated speaker, and feedback controller with disturbance $D_{2}$
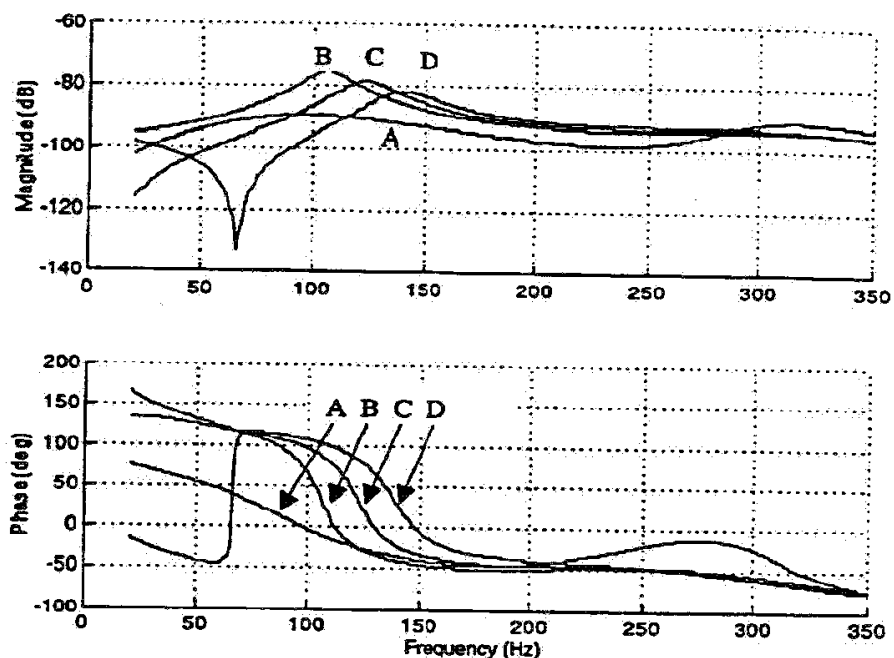

Figure 10. Frequency response of the $Q_{1} / P_{1}$ transfer function with the resonator, compensated speaker and feedback controller for four cases with gains shown in Table 3

modeled. Figure 9 shows the block diagram of the resonator and compensated speaker and controller with a disturbance $D_{2}$.

The closed-loop, compensated system response can now be simulated to verify that the acoustic resonance of the system can be modified by the feedback controller. Although the compensator was added to force the actuator response to approach the ideal actuator model, Birdsong (1999) showed that the actuator dynamics could not be sufficiently minimized by this technique. While this complicates the controller design somewhat, the simple PI controller could still be successfully used. A model based, empirical controller design (Birdsong, 1999) was used to find gains that produced the desired response. Figure 10 shows the frequency response of the $Q_{1} / P_{1}$ transfer function for four cases with the controller gains given in Table 3. These results show that the compensated actuator successfully implements the closed-loop control. The controller moves the frequency of the peak and zero phase to 106, 123, and $139 \mathrm{~Hz}$ Note that the maximum amplitude of each resonant peak decreases with frequency. Also, the magnitude of graph D falls below the open-loop graph A at $65 \mathrm{~Hz}$. This shows that if the SHR is misstuned then the closed-loop response can be worse than the openloop. Nonetheless, with proper tuning, the compensated actuator and SHR behave as a tunable acoustic resonator. 

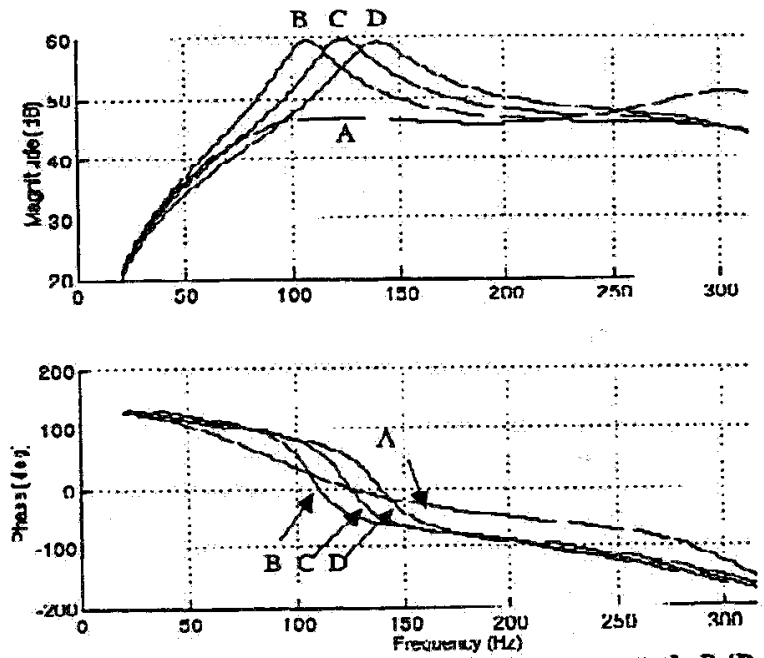

Figure 11. Frequency response of the compensated $P_{2} / D_{1}$ transfer function: A: open-loop, B, C, and D closed-loop with gains selected (Table 3 ) to increase resonant peak and move resonant frequency

Table 3. Compensator \& Controller Gains for Fig. 10 \& 11

\begin{tabular}{|c|c|c|c|c|}
\hline Curves & A & B & C & D \\
\hline Compensator In / Out & In & In & In & In \\
\hline$K_{\text {amp }}$ & 30 & 30 & 30 & 30 \\
\hline$K_{P}$ & 0 & -0.81 & -0.9 & -0.9 \\
\hline$K_{I}$ & 0 & -250 & 0 & 250 \\
\hline Resonant Freq. (Hz) & 102 & 106 & 123 & 139 \\
\hline Percent Damping & 50 & 10 & 10 & 10 \\
\hline
\end{tabular}

Another transfer function $P_{2} / D_{1}$, is of interest in this system because it is used to compare the model and experimental results. Although the $Q_{1} / P_{1}$ transfer function is the key to the effectiveness of the device for noise control, it is difficult to measure experimentally. The volume velocity flow, $Q_{1}$, is a zero mean oscillating air velocity. A laser velocity anemometer can be used to make such a measurement, but this is an expensive and complex device. Instead, the $P_{2} / D_{1}$ transfer function can be examined to observe the resonant frequency and damping. Figure 11 shows the closed-loop $P_{2} / D_{1}$ transfer function with the gains in Table 3. The model based empirical controller design finds gains, $K_{P}$ and $K_{I}$, that produce resonant peaks with constant magnitude in the $P_{2} / D_{1}$ transfer function. Note this results in resonant peaks that decrease in amplitude with increasing frequency in the $Q_{1} / P_{1}$ transfer function (Figure 10). As before, the magnitude attains a peak and the phase crosses zero at the resonant frequency.

One undesirable feature of the speaker compensator is that it introduces noise into the actuator output. This is because it uses the voltage from the speaker secondary coil to estimate the secondary coil current. The secondary coil voltage is a low level signal with a low signal to noise ratio. The noise is amplified by the compensator gain $K_{a m p}$. This can be analyzed by modeling a disturbance $D_{3}$ input to the secondary coil current. The frequency response of the transfer function for $P_{2} / D_{3}$ is shown in Figure 12 for the compensator and controller settings in Table 3 . These results indicate that random noise in the frequency range of $50-$ $400 \mathrm{~Hz}$ will be injected into the actuator output. Although the signal to noise ratio could be increased by increasing the number of windings on the secondary coil, this was not done in this work.

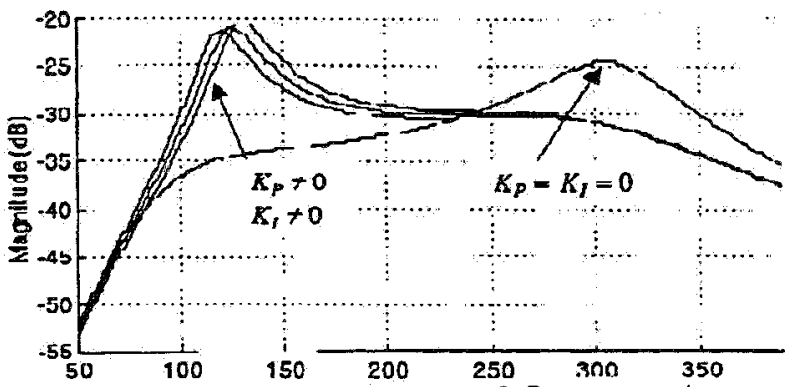

Figure 12. Frequency response of $P_{2}$ to current sensor disturbance for resonator, compensated speaker and feedback controller model with gains from Table 3

Resonator, Speaker, \& Controller - No Compensation

A final model is examined which includes the resonator, uncompensated speaker, and feedback controller, to compare the compensated system with the uncompensated system. The block diagram for the speaker compensation includes a switch that removes the local feedback compensation from the loop. The model was assembled with the same components as the previous model, but with the local feedback compensation removed. The frequency response of the $P_{1} / Q_{1}$ transfer function for the resonator, uncompensated speaker, and feedback controller was then simulated and shown in Figure 13. It was found that the PI controller was not able to amplify and move the low frequency resonance $(80 \mathrm{~Hz})$. Instead the high frequency resonance at 290 $\mathrm{Hz}$ was amplified and moved by the application of the controller. Note that a peak in magnitude is attained and a zero phase occurs at the different resonant frequency. This verifies that the uncompensated actuator can be used in the SHR system. The frequency response of the $P_{2} / D_{1}$ transfer function was also simulated, shown in Figure 14 for comparison with experimental results.

The controller gains were selected to increase and decrease the resonance approximately $10 \%$ from the nominal value while maintaining a damping ratio of $10 \%$. Note considerably different gains are needed to obtain these results as compared with the compensated system. The integral gain, $K_{I}$, is much larger than before, 1,600 to 12,800 compared to -100 to 100 for the uncompensated system. There are several explanations for this. A value of $K_{\text {amp }}=1$ was used in the uncompensated system compared with $K_{\text {emp }}=30$ in the compensated system. Also, the integral of the pressure signal decreases with frequency requiring a gain three times as large to effect the resonance at $300 \mathrm{~Hz}$ as one at $100 \mathrm{~Hz}$. Note too the trends of the gains are very different than the uncompensated system. A more complete discussion of the mapping of the controller gains to the resonant frequency and damping is beyond the scope of this article and is given in Birdsong, (1999). Finally, note the peak magnitudes of graphs B, $C$ and $D$ in Figure 14 are approximately $5 \mathrm{~dB}$ less than those with the compensated speaker model, Figure 11. Increasing the peak magnitude further would require reducing the system damping, which would lead to reducing the stability margin of the system.

These results indicate that the SHR with the uncompensated actuator is capable of producing an electronically tuned acoustic resonator. No significant noise is introduced into the system because the compensator is not present. Also the nominal resonant frequency is increased significantly $(300 \mathrm{~Hz})$ compared to the compensated system $(130 \mathrm{~Hz})$. However, the maximum magnitude of the uncompensated system is less than the compensated system. 

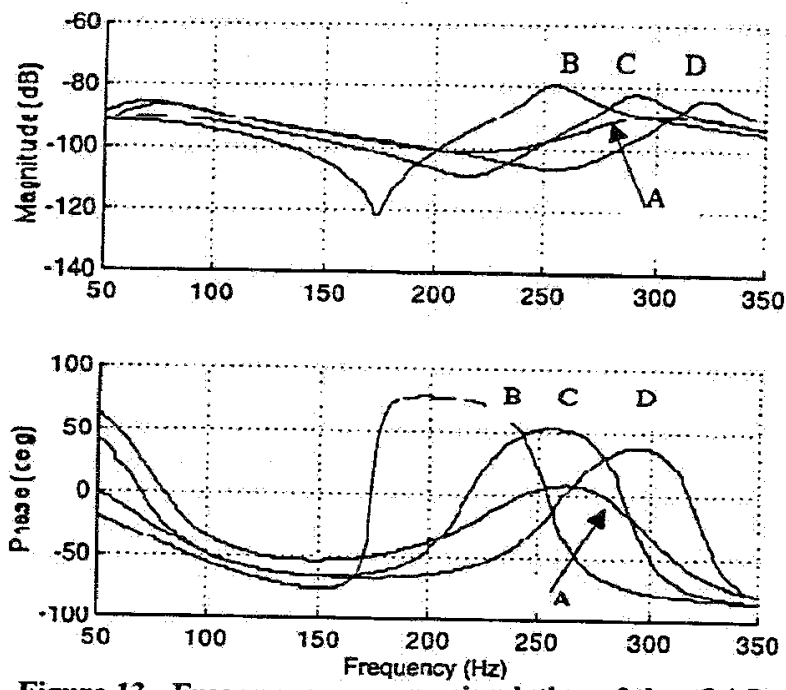

Figure 13. Frequency response simulation of the $Q_{1} / P_{1}$ transfer function with the resonator, uncompensated speaker, and feedback controller coupled model with controller gains from Table 4
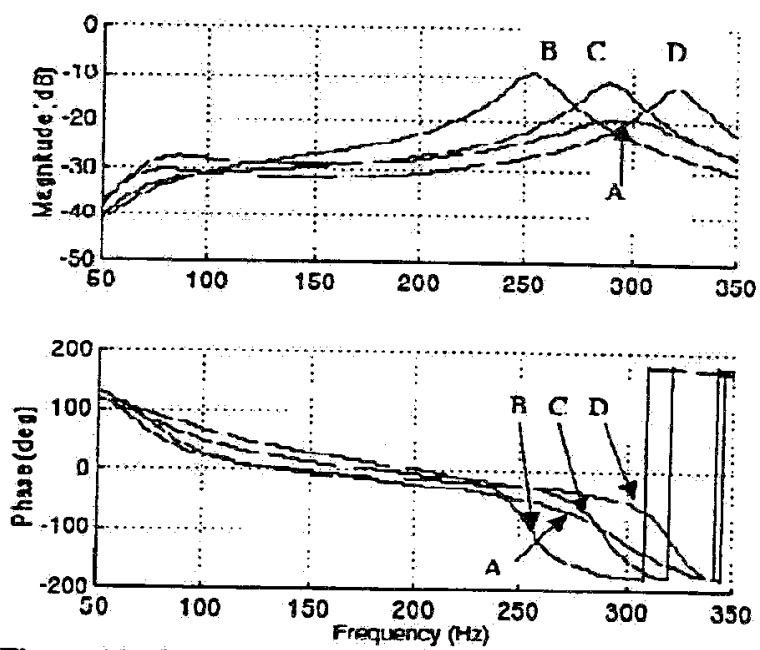

Figure 14. Frequency response simulation for the $P_{2} / D_{1}$ transfer function with the resonator, uncompensated speaker, and feedback controller coupled model with controller gains from Table 4

Table 4. Controller Gains For Figures 13 and 14

\begin{tabular}{|l|c|l|l|l|}
\hline Curves & A & B & C & D \\
\hline$K_{P}$ & 0 & -10 & 0 & 10 \\
\hline$K_{I}$ & 0 & 12,800 & 9,600 & 1,600 \\
\hline Resonant Freq. (Hz) & 298 & 254 & 290 & 322 \\
\hline Percent Damping & 50 & 10 & 10 & 10 \\
\hline
\end{tabular}

A comparison of the compensated and uncompensated actuators suggest that each have use for different applications. The larger magnitude response of the compensated actuator suggests that it is more effective in controlling noise in a narrow frequency band. That is, the compensated SHR will reflect more narrow frequency sound in a duct than the uncompensated SHR. It, therefore, may be more effective when the objective is to minimize narrow band pressure oscillations. However, the compensated SHR adds broadband noise to the system thus degrading some of the noise reduction that is sought. The

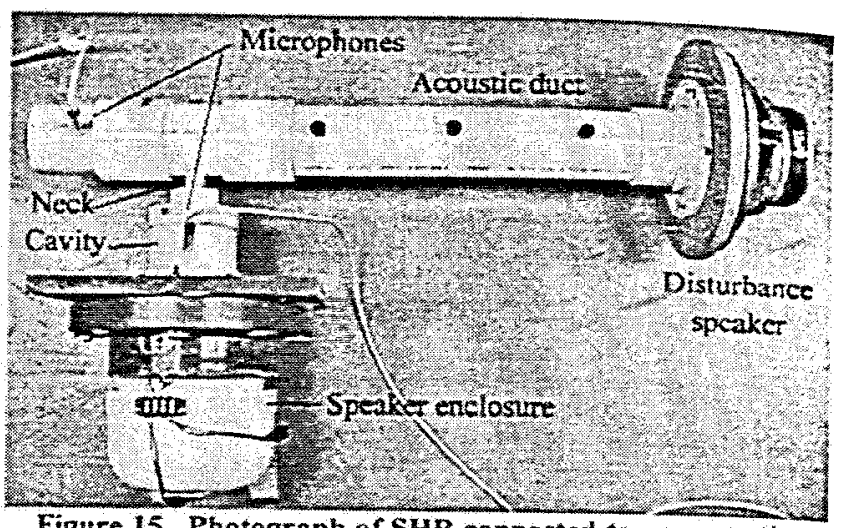

Figure 15. Photograph of SHR connected to an acoustic duct with a second audio speaker to inject noise

uncompensated SHR does not reflect as much noise in a duct, but does not add as much noise to the system. It, therefore, may be more effective when the objective is to improve overall sound quality.

\section{EXPERIMENTAL VALIDATION}

An experimental apparatus was constructed to validate the theoretical model and to demonstrate the noise reduction capability of the device. Figure 15 shows a photograph of the SHR connected to an acoustic duct with a disturbance speaker. The SHR consisted of two components: a Helmholtz resonator cavity and a microphone-controller-compensated actuator system (Figure 16). A cylindrical Helmholtz resonator cavity was constructed from PVC with diameter $0.075 \mathrm{~m}$ and length $0.15 \mathrm{~m}$. A cylindrical neck with diameter $0.018 \mathrm{~m}$ and length $0.01 \mathrm{~m}$ was fitted on one face of the cavity. The microphone-compensated actuator system consisted of a half-inch B\&K type 4155 microphone sealed through the wall of the cavity. A D-Space Model \#1102 floating point digital signal processor (DSP) was used to implement the speaker compensation, and an acoustic actuator was sealed in the opposite face of the cavity. A DSP sampling rate of $5 \mathrm{kHz}$ was used for all experiments. The actuator consisted of a 6 inch dual voice coil speaker with local compensation (Birdsong and Radcliffe, 1999) to improve the speaker velocity response.

\section{Compensated Actuator Results}

A speaker velocity estimator (Birdsong and Radlciffe, 1999; Radcliffe and Gogate, 1996) was created by combining the voltage in the secondary coil with the current in the primary coil. The controller was added to the system and gains were found to amplify the resonant peak and shift the resonant frequency. The gains were found using a model based empirical technique (Birdsong, 1999) that produced a response with different resonant frequencies and constant peak amplitude. Figure 17 shows the results for 4 experiments, curves labeled $A, B, C$, and $D$ that were generated using the controller gains in Table 5 . These gains reduced the percent damping from $50 \%$ with $K_{J}=K_{p}=0$ to $5 \%$ and shifted the peak from $130 \mathrm{~Hz}$ to $100 \mathrm{~Hz}$ and $170 \mathrm{~Hz}$.

The next experiment demonstrates noise reduction in a duct and the introduction of random noise into the system by the actuator compensator. In this experiment the SHR was attached to an acoustic duct and a pure tone of $130 \mathrm{~Hz}$ was injected into one end of the duct by a second audio speaker (Figure 18). The sound pressure level (SPL) was then recorded at the duct end with the SHR in two configurations: first, with the uncompensated openloop system, then with the compensated, closed-loop system with the controller gains selected to tune the system to $130 \mathrm{~Hz}$. Figure 19 shows both spectra. With the uncompensated, open-loop 


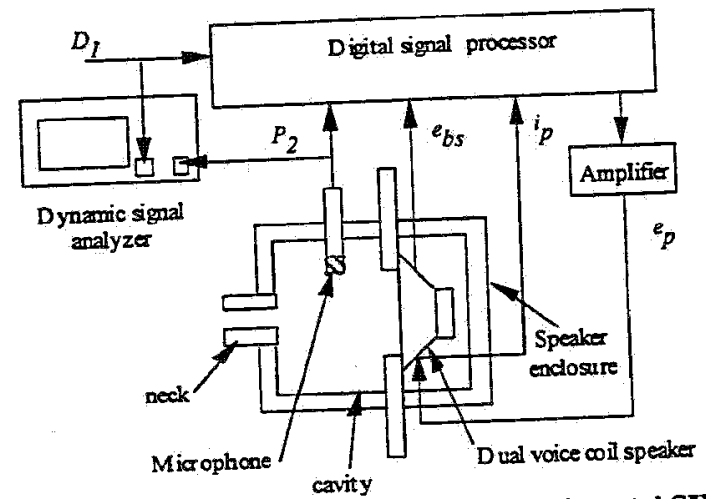

Figure 16. Schematic diagram of experimental SHR apparatus

system, the pure tone appears as a $117 \mathrm{~dB}$ spike in the spectrum at $130 \mathrm{~Hz}$ (dashed line). Other harmonics at 60 and 180 are tistortion in the disturbance speaker. With the compensated, closed-loop system, the spectrum (solid line), compensated tone at $130 \mathrm{~Hz}$ is reduced dramatically to $85 \mathrm{~dB}$, shows the tone as 130 noise reduction. Nonetheless, significant background noise is introduced by the closed-loop actuator in a broad band between 60 and $200 \mathrm{~Hz}$. This sound is below $80 \mathrm{~dB}$, but becomes significant since the disturbance has been reduced to $85 \mathrm{~dB}$. The shape of the broadband noise is similar to the $P_{2} / D_{2}$ transfer function result predicted by the model in Figure 12. It is attributed to random electrical noise introduced in the speaker secondary coil voltage and magnified by the compensation amplifier $K_{a m p}$. The overall sound pressure level was also recorded at the open duct end using a B\&K sound level meter. With the uncompensated, open-loop system, the overall sound level was $118 \mathrm{~dB}$, and with the compensated closed-loop prstem the overall sound pressure level was $100 \mathrm{~dB}$, representing $18 \mathrm{AB}$ of overall SPL noise reduction.

Uncompensated Actuator Results

The previous experiments were repeated with the actuator in the uncompensated configuration. Figure 20 shows the uncompensated closed-loop $F_{2} / D_{1}$ response without the duct with gains from Table 6 . As predicted by the model, the ine lower frequency. uncompensated actuator do occurs at a higher frequency near 240 Instead the only resonance occurs a $20 \mathrm{~dB}$ $\mathrm{Hz}$. The controller was able to amplify the $240 \mathrm{~Hz}$ with gains $K_{P}=K_{\text {, }}$

and shift the resonant frequency from $240 \mathrm{~Hz}$
$=0$ higher and lower in frequency by $25 \mathrm{~Hz}$.

Table 5. Controller gains used in Figure 17

\begin{tabular}{|l|l|l|l|l|}
\hline Graph & $\mathrm{A}$ & $\mathrm{B}$ & $\mathrm{C}$ & $\mathrm{D}$ \\
\hline$K_{P}$ & 0 & -0.34 & -0.34 & -0.24 \\
\hline$K_{I}$ & 0 & -120 & 30 & 250 \\
\hline$K_{a m p}$ & 30 & 30 & 30 & 30 \\
\hline Resonant Frequency (Hz) & 130 & 100 & 130 & 170 \\
\hline Percent Damping & 50 & 5 & 5 & 5 \\
\hline
\end{tabular}
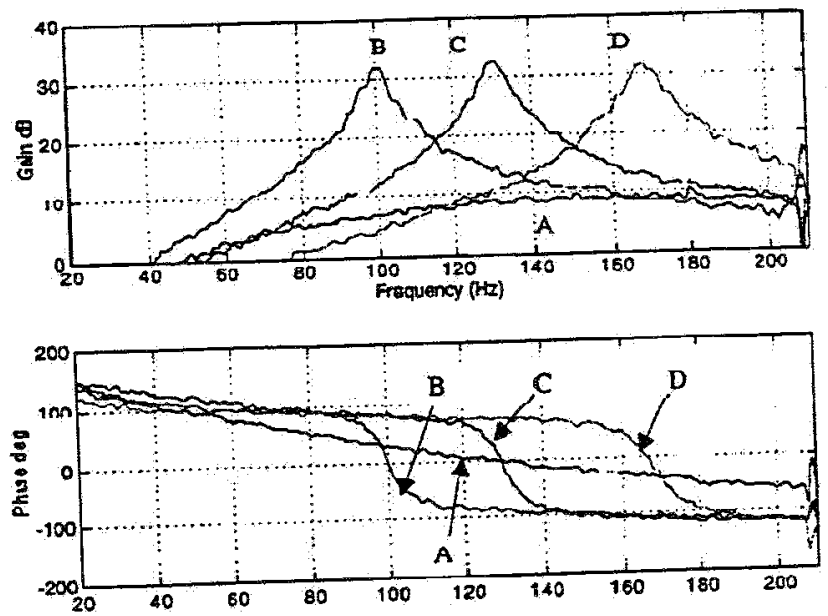

Figure 17. Experimental closed-loop frequency response of coupled system with compensated actuator showing that controller amplifies the peak magnitude and moves the resonant frequency

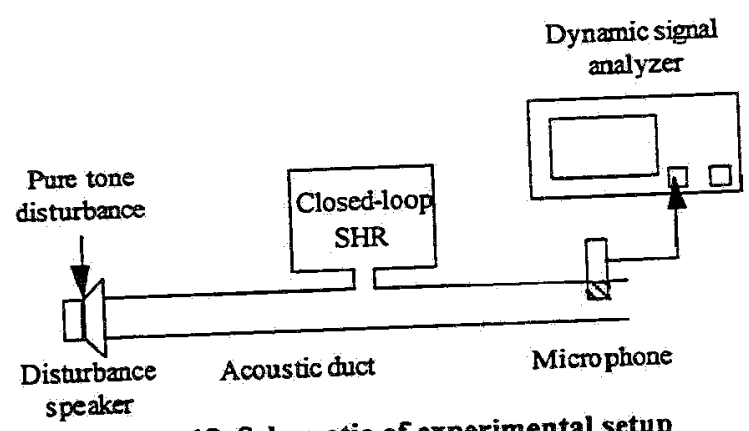

Figure 18. Schematic of experimental setup

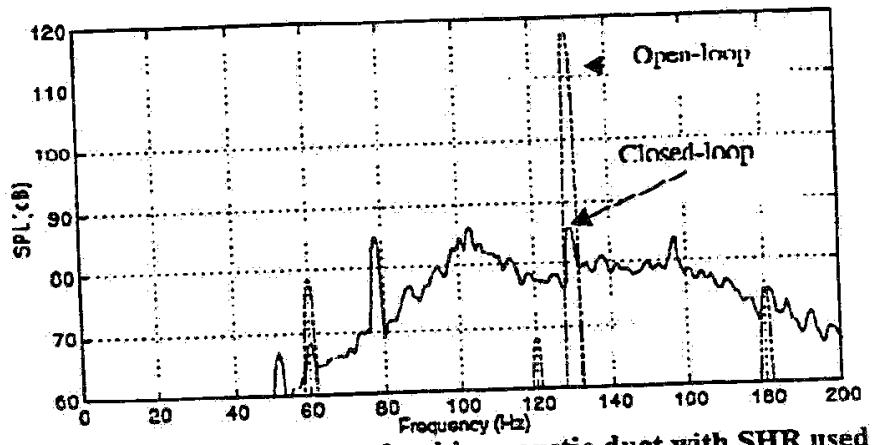

Figure 19. Sound pressure level in acoustic duct with SHR used to reduce pure tone disturbance, dashed line: compensator and controller out of the loop, solid line compensator and controll in the loop with controller gains set to tune SHe 

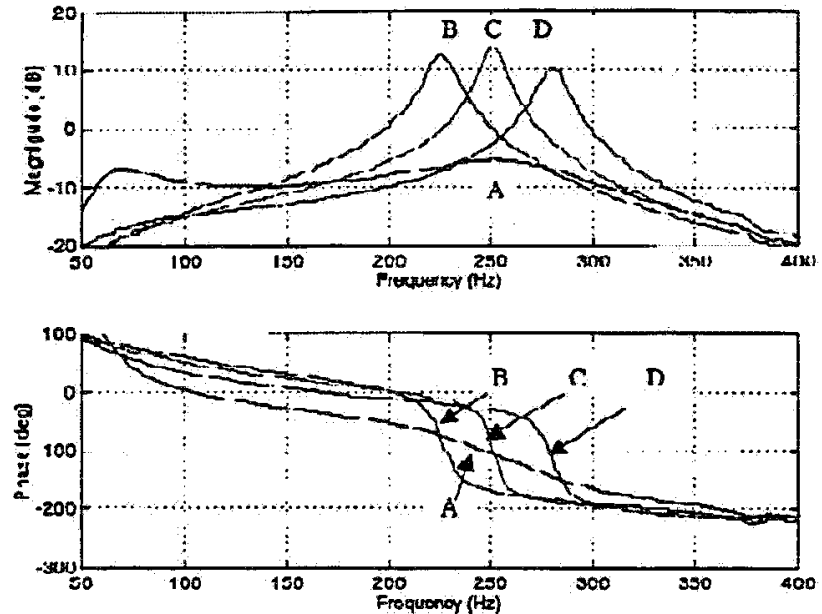

Figure 20. Experimental frequency response of closed-loop SHR with uncompensated actuator

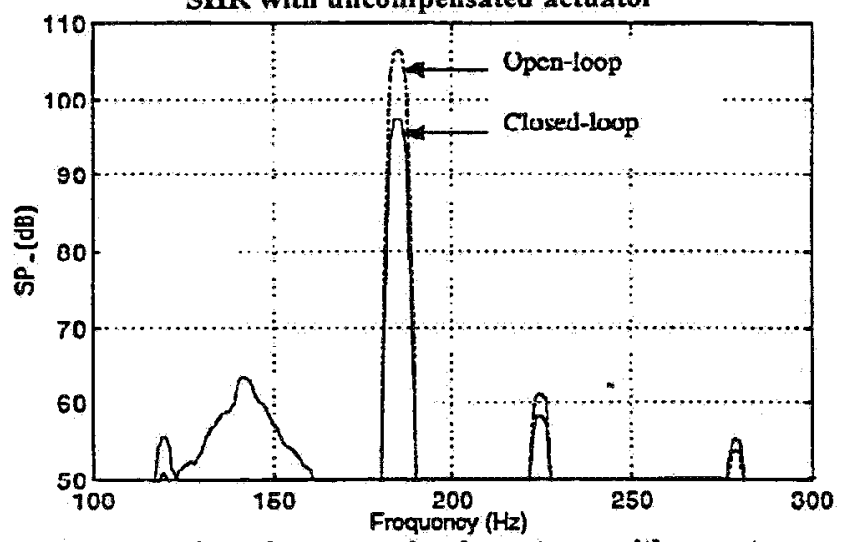

Figure 21. Sound pressure level spectrum with pure tone disturbance at $185 \mathrm{~Hz}$ with open and closed-loop SHR and uncompensated actuator

Table 6. Controller gains used for Figure 20

\begin{tabular}{|l|l|l|l|l|}
\hline Graph & A & B & C & D \\
\hline$K_{P}$ & 0 & 0 & 1.0 & 2.0 \\
\hline$K_{I}$ & 0 & 2520 & 2520 & 1740 \\
\hline$K_{a m p}$ & 1 & 1 & 1 & 1 \\
\hline Resonant Freguency (Hz) & 250 & 225 & 250 & 280 \\
\hline Percent Damping & 50 & 6 & 6 & 6 \\
\hline
\end{tabular}

The last experiment shows that the SHR with the uncompensated actuator reduces noise in a duct without introducing significant random noise into the system. The SHR and duct setup (Figure 18) was repeated with the uncompensated actuator and SHR. A $185 \mathrm{~Hz}$ pure tone was injected into the duct end by the second audio speaker. Figure 21 shows the SPL spectrum recorded at the duct end with open-loop (dashed line), then closed-loop with gains set to tune the system to match the noise frequency (solid line). With the open-loop system, the peak SPL is $107 \mathrm{~dB}$ at $185 \mathrm{~Hz}$. With the closed-loop system, the noise level is reduced to $98 \mathrm{~dB}$, representing a $9 \mathrm{~dB}$ noise reduction. The background noise level is below $60 \mathrm{~dB}$ indicating that the uncompensated actuator does not introduce significant noise to the system. The overall SPL measured with a sound level meter showed identical results $(9 \mathrm{~dB}$ noise reduction) indicating that in both open and closed-loop settings the noise is dominated by the narrow band tone at $185 \mathrm{~Hz}$

\section{CONCLUSIONS}

The actuator is a critical component in the implementation of the SHR. Compensated and uncompensated actuators were presented. Both were shown by analytical model and experimental results to be effective in the SHR. Both uncompensated and compensated actuators introduced significant dynamics into the system, requiring modification of the feedback controller design. However the compensated actuator was found to introduce random noise, degrading the overall SPL noise reduction. The uncompensated actuator did not introduce noise into the system, but could not generate as strong a resonance as the compensated actuator. It also had a higher resonant frequency. These conclusions lead to a criterion for choosing which actuator is optimal for different applications. For applications where a narrow band disturbance must be minimized without concern for the sound quality, the compensated actuator is superior. Altematively, if sound quality is of concern, then the random noise introduced by the compensation may be objectionable even though the overall SPL is higher. In this case, the uncompensated actuator may be a better choice.

\section{REFERENCES}

Birdsong, C. B., 1999, "A Semi-Active Helmholtz Resonator," Ph.D. Dissertation, Michigan State University, East Lansing, Michigan.

Birdsong, C. B., and Radcliffe, C. J., 1999, "A Compensated Acoustic Actuator for Systems with Strong Dynamic Pressure Coupling," Journal of Vibrations and Acoustics, Vol. 121, pp. 8994.

Birdsong, C., 1996, "A compensated Actuator for an Acoustic Duct," Masters Thesis, Michigan State University.

Harwood, H.D., 1974, "Motional Feedback in Loudspeakers,"

Wireless World, 80, pp. 51-52.

Holdaway, H.W., 1963, "Design of Velocity Feedback Transducer Systems for Stable Low-Frequency Behavior," IEEE Transactions, AU-11, pp. 155-173.

Klaassen, J.A., de Koning, S.H., 1968, "Motional Feedback with Loudspeakers," Philips Technical Review, 29, No. 5, pp. 148157.

Phillips, C., and Harbor, R., 1996,"Feedback Control

Systems," Prentice-Hall, New Jersey.

Pierce, Allan D., 1981, "Acoustics : an introduction to its physical principles and applications," New York : McGraw-Hill Book Co.

Radeliffe C. J., and Gogate, S. D., 1996, "Velocity Feedback Compensation of Electromechanical Speakers for Acoustic Applications," Intemational Federation of Automatic Control, Triennial World Congress, July.

Radcliffe C.J., and Gogate S.D., Hall G., 1994, "Development of an Active Acoustic Sink (AAS) for Noise Control

Applications," Active Control of Vibrations and Noise, ASME.

Radcliffe, C. J., and Gogate, S. D., 1992, "Identification and

Modeling Speaker Dynamics for Acoustic Control Applications," ASME Symposium on Active Control of Noise and Vibration.

Tang, P. K., and Sirignano, W. A., 1973, "Theory of a

Generalized Helmholtz Resonator," Journal of Sound and

Vibration, vol. 26 (2), pp. 247-262.

Tanner, R.L., 1951, "Improving Loudspeaker Response with Motional Feedback," Electronics, 24, No. 3 Inc 